$48 \mid 2017$

L'art de mentir

\title{
In memoriam Françoise Aubin (1932-2017)
}

Isabelle Charleux et Roberte Hamayon

\section{(2) OpenEdition}

\section{Journals}

Édition électronique

URL : https://journals.openedition.org/emscat/2964

DOI : 10.4000/emscat.2964

ISSN : 2101-0013

Éditeur

Centre d'Etudes Mongoles \& Sibériennes / École Pratique des Hautes Études

\section{Référence électronique}

Isabelle Charleux et Roberte Hamayon, «In memoriam Françoise Aubin (1932-2017) », Études mongoles et sibériennes, centrasiatiques et tibétaines [En ligne], 48 | 2017, mis en ligne le 05 décembre 2017, consulté le 13 juillet 2021. URL : http://journals.openedition.org/emscat/2964 ; DOI : https:// doi.org/10.4000/emscat.2964

Ce document a été généré automatiquement le 13 juillet 2021.

(C) Tous droits réservés 


\title{
In memoriam Françoise Aubin (1932-2017)
}

\author{
Isabelle Charleux et Roberte Hamayon
}

\section{NOTE DE L'AUTEUR}

Ce texte reprend en grande partie la préface rédigée par Denise Aigle, Isabelle Charleux, Vincent Goossaert et Roberte Hamayon pour la publication d'un volume en l'honneur de Françoise Aubin dans une institution qu'elle a fréquentée toute sa vie, Monumenta Serica : Miscellanea Asiatica. Festschrift Françoise Aubin (Sankt Augustin, Monumenta Serica Monograph Series LXI, 2011). Que l'éditeur soit remercié de nous autoriser à la reproduire ici.

1 C'est avec une grande tristesse que nous avons appris la disparition de notre collègue et amie Françoise Aubin, directeur de recherche honoraire au CNRS, le 10 juillet 2017, à l'âge de 85 ans.

2 Françoise Aubin était une grande figure de notre domaine. Juriste de formation, orientaliste par passion, et humaniste par nature, elle a su toute sa vie allier l'érudition et l'intelligence des faits sociaux. Sa soif de connaissances et sa générosité intellectuelle nous manqueront.

3 Françoise Aubin a accompagné la vie de notre revue pendant de longues années. Membre de son Comité de rédaction dès ses débuts au Laboratoire d'ethnologie et de sociologie comparative de Paris-X Nanterre, en 1970, elle a suivi ses transformations successives. Les Cahiers d'études mongoles lancés dans l'enthousiasme, grâce à la collaboration des étudiants de mongol à l'École des Langues Orientales, étaient devenus la revue Études mongoles et sibériennes domiciliée à l'École Pratique des Hautes Études lorsque Françoise a mis fin à ses activités éditoriales en 2006. Françoise a fait bénéficier la revue de ses larges compétences linguistiques, de ses conseils de lecture, de ses connaissances factuelles et de ses domaines d'intérêt comme de son souci de rigueur, d'exactitude et de précision. Elle a personnellement contribué à son essor par la 
richesse, l'intérêt et la qualité scientifique de ses contributions comme par sa passion de faire connaître : articles, comptes rendus, nécrologies, traduction et adaptation de travaux de chercheurs étrangers ${ }^{1}$.

4 Lorsque Françoise Aubin s'inscrit à l'École des Langues Orientales à la sortie de ses études secondaires, elle y apprend le russe, le japonais et le chinois. Le mongol n'y étant pas enseigné, elle se familiarise avec cette langue aux séminaires de Louis Hambis à l'École Pratique des Hautes Études (IV section), et auprès des mongolisants de Budapest (Louis Ligeti, György Kara et András Róna-Tas) où elle séjourne en 1962 et 1965. Lorsque s'ouvrent les relations diplomatiques entre la France et la Mongolie en 1966, elle donne son accord pour créer un enseignement du mongol aux «Langues'O ». Atteinte par la maladie de Guillain-Barré, elle ne peut assurer que quelques cours de civilisation au tout début de cet enseignement en 1968.

5 L'ampleur de ses compétences disciplinaires est tout aussi impressionnante que celle de ses compétences linguistiques. Formée initialement en sciences juridiques, elle se révèle vite une historienne accomplie, comme le montre la thèse qu'elle a soutenue à la faculté de droit de Paris sous le titre "Les soulèvements populaires en Chine du Nord entre 1214 et 1230 ", qui reçoit le prix de la meilleure thèse en 1965. À travers ce début de XIII ${ }^{\mathrm{e}}$ siècle qui voit l'émergence de Chinggis Khan et la formation de la nation mongole, Françoise étudie l'impact, sur la société chinoise rurale, d'un nouveau type de pouvoir. Loin de limiter son intérêt à cette période, Françoise Aubin s'interroge aussi sur le devenir contemporain de ce peuple nomade. C'est ainsi qu'elle met à profit la présence en France d'une petite colonie de Kalmouks émigrés pour mener une enquête approfondie sur son histoire et ses particularités culturelles, qui reste de nos jours encore une source unique sur les Kalmouks de France (1966). La signature d'un protocole d'échange entre le CNRS et l'Académie des sciences de Mongolie lui permet de se rendre dans ce pays en 1966 puis à nouveau en 1967 : elle y sera le premier chercheur ressortissant d'un pays capitaliste accueilli par l'Académie. Les enquêtes de terrain qu'elle réalise dans ce pays révèlent chez elle des talents d'ethnologue et de sociologue à l'aise tant pour traiter de questions techniques (concernant entre autres l'élevage et l'usage des chevaux) que pour aborder les domaines politique et religieux, ce qui autorise à classer son œuvre aussi dans le champ des sciences humaines au sens large. Ainsi est-ce d'une double carrière qu'il faut ici faire état, en orientalisme et en sciences humaines. Si l'avancée de la maladie lui rend définitivement pénible tout déplacement, lui interdisant tout travail de terrain, elle ne fait qu'exacerber sa sensibilité anthropologique, en permanence à l'affut dans la lecture des textes et les discussions avec les collègues.

De ses missions en Mongolie sous le régime communiste, Françoise Aubin rapporte des études pionnières aussi bien sur l'expérience de la collectivisation et l'organisation des coopératives rurales (1967) que sur l'utilisation des festivités et des commémorations pour faire passer des messages idéologiques. Son article sur «le statut de l'enfant » illustre parfaitement l'alliance heureuse qu'elle réalise entre formation juridique, maitrise des sources et sens de l'anthropologie ; depuis sa publication en 1975, il a été exploité par toutes les générations de mongolisants. Juste après la fin du régime communiste, elle est l'une des premières à percevoir, grâce à sa lecture perspicace de la presse, l'exploitation nationale, voire nationaliste, de la figure de Chinggis Khan et les premières tentatives d'établir les bases de ce qui allait bientôt devenir un véritable culte en son honneur (1993). 
7 Tout en maintenant sans aucune relâche son intérêt pour la Mongolie (publiant, participant à des jurys, et suivant l'actualité scientifique et politique), Françoise défriche de nouveaux thèmes de recherche dans les années 1970, 80 et 90, faisant le meilleur usage de la liberté permise par le $\mathrm{CNRS}^{2}$. Travaillant dans le prestigieux cadre du CERI, elle défriche un à un les différents champs qui aujourd'hui l'honorent comme une personnalité fondatrice. Le premier d'entre eux, chronologiquement, est l'histoire des missions en Extrême-Orient, et en particulier du catholicisme de langue chinoise (même si, contrairement à la plupart des spécialistes du catholicisme chinois elle écrit aussi sur le protestantisme). Tout en gardant une posture distincte de l'histoire « interne » des missions, Françoise entretient avec ce monde de très bons rapports d'intelligence réciproque. Il en est ainsi en particulier avec les missions de Scheut (CICM), congrégation essentiellement flamande implantée en Mongolie-Intérieure jusqu'en 1949 et qui a fourni quelques-uns des plus grands mongolisants. Le travail que mène Françoise dans les archives de Scheut (des manuscrits allusifs en flamand d'église) force l'admiration de ceux qui l'ont vu de près. En outre, à l'instar des missionnaires éclairés dont elle analyse et prolonge les travaux, Françoise est sensible à l'ensemble du paysage culturel et religieux, et sa culture sur le confucianisme, le bouddhisme et le taoïsme va bien au-delà du simple besoin de ses propres recherches.

8 Le travail que Françoise Aubin effectue sur les efforts missionnaires envers les musulmans de Chine la mène à se pencher sur l'islam. Elle forme de jeunes chercheurs lors de séminaires, publie des synthèses remarquables et des études originales, notamment sur les confréries soufies. La grande portée de ces travaux, reconnus par des invitations à Harvard et autres grandes universités, s'appuie sur une vision d'ensemble, et sur la maîtrise des langues, depuis le turc jusqu'au japonais, qui permet de situer l'islam de Chine dans son vaste contexte et de retracer ses liens, avec le monde arabe comme avec le monde persan - lequel a été naguère si brillamment étudié par le regretté Jean Aubin, qui fut son époux et compagnon intellectuel jusqu'à son décès en 1998, et dont Françoise assura vaillamment la publication des œuvres posthumes.

9 Si les travaux de Françoise Aubin ont eu un tel écho pour tant de chercheurs dans des domaines si divers, ce n'est pas seulement en raison de l'abondance et de la qualité des données qu'elle met à leur disposition - aussi exhaustives que faire se peut et toujours minutieusement vérifiées -, ni en raison de l'agrément de son écriture, qu'animent tour à tour ou tout à la fois l'agilité d'esprit, l'enthousiasme ou l'humour. C'est aussi et peutêtre surtout en raison du sens qu'a Françoise Aubin de ce qui est "pertinent » pour saisir la portée d'un événement historique ou la spécificité d'une culture; elle sait percevoir dans une situation ou une institution, le détail significatif, dans un mobilier ou un équipement, l'objet révélateur qui, replacés dans le contexte global, jetteront un éclairage neuf sur la société, sa culture ou son histoire.

10 C'est ce qu'illustrent plusieurs de ses travaux qui, consacrés à des études ponctuelles d'objets matériels, le cadenas par exemple, ou de certains aspects culturels méconnus de la vie nomade, débouchent sur des aperçus de portée générale. Ainsi, l'étude des «mesures manuelles et par référence au corps » donne-t-elle à voir l'existence d'un ensemble cohérent de concepts et de gestes que les nomades mongols ont constitué un véritable système à valeur juridique pour réguler les échanges commerciaux en leur sein, mais qu'ils utilisent aussi dans la vie sociale, tant pour calculer les degrés de parenté que pour évaluer des objets d'usage quotidien (1970). 
11 De ce point de vue, l'article consacré à la selle chez les Mongols sous le titre « le folklore comme mass media » (1986) est peut-être plus exemplaire encore. La selle du nomade mongol type, membre de collectivité rurale, dans les années 1970 et 1980, est en effet «lourdement ornée d'argent, d'ivoire, de velours ». Or avant l'avènement du régime communiste, elle ne l'était pas; si elle était un bien personnel, elle n'était pas pour autant un bien précieux dans le budget du nomade moyen, et ne faisait pas l'objet d'attentions rituelles. Proverbes et dictons, nombreux à mettre la selle en parallèle avec la femme, témoignent d'une frappante «ambiguïté sentimentale» à son égard: elle peut être utile, à condition qu'elle soit soumise. Certes, l'amélioration des revenus a sa part dans ce changement, souligne Françoise Aubin avant d'évoquer, d'une plume allègre, une autre raison: "le transfert, vers la selle du mari d'un luxe de parade exhibé, précédemment, dans la coiffure de l'épouse ». Un souci d'égalité générale avait en effet conduit la jeune République populaire à interdire les parures féminines qui portaient jadis les distinctions de fortune et d'appartenance ethnique. Comment mieux prouver, conclut l'auteur, l'utilité de la dimension diachronique dans l'étude de ce qui est présenté comme "tradition populaire », comment mieux illustrer l'usage d'un objet tel que la selle comme «support de discours politique»? Et comment mieux démontrer, ajouterons-nous, que même un objet d'intérêt apparemment mineur peut donner lieu à une recherche large et féconde?

Il importe de s'arrêter, dans le rappel de cette carrière d'une exceptionnelle longévité puisqu'elle s'est déroulée sur plus de cinquante ans, sans rupture d'activité de publication, sur l'œuvre de recension accomplie par Françoise Aubin. Animée d'une curiosité inlassable, elle cherche à lire la quasi-totalité des publications relatives à ses divers domaines de recherche. Aussi est-ce une œuvre inégalable qu'elle a accomplie dans ce domaine trop souvent négligé, une œuvre d'une rare qualité grâce à l'ampleur de ses connaissances et de sa capacité de synthèse. Sa bibliographie compte en effet les comptes rendus de plus de mille ouvrages qu'elle a recensés ${ }^{3}$. Elle a ainsi fait connaître au public occidental de nombreux ouvrages en langues souvent difficiles d'accès, sachant immédiatement en saisir l'intérêt et y réagir avec un jugement très sûr, en mettre en lumière les points forts, et en caractériser l'apport scientifique en quelques lignes toujours claires, pour inviter, selon les cas, à lire l'ouvrage ou à s'en abstenir.

13 En signe de reconnaissance pour sa contribution à la recherche dans le domaine mongol, Monsieur M. Batsaikhan, ambassadeur de Mongolie en France a remis la plus haute distinction de Mongolie, l'Etoile Polaire (Altan gadas), à Françoise Aubin, entourée de ses amis, de ses collègues et de sa famille, dans sa maison du Parc de Séronne, le 18 février $2017^{4}$.

Enfin, si Françoise, comme savant, a mérité le respect de ses collègues par ses publications, elle a gagné l'affection de ceux qui l'ont fréquentée de près par la façon profondément humaniste dont elle vivait son métier et son rapport aux autres, en particulier aux étudiants et jeunes collègues. Son réseau de relations dans le monde entier est solidement bâti sur une grande fidélité dans ses amitiés. Soulignons encore sa générosité à mettre sa bibliothèque et son savoir à la disposition des étudiants, dont elle suivait et soutenait la carrière, corrigeant inlassablement leurs articles. Des séjours dans sa demeure de l'Anjou ont permis à de nombreux jeunes chercheurs de prendre la mesure de ce qu'est l'érudition, et de la masse de travail minutieux qu'elle requiert, mais aussi de partir rassérénés (et bien nourris) avec des notes, des photocopies, des conseils judicieux, des pistes nouvelles. Si des savants de tous les continents sont venus 
en Anjou dévorer tant sa bibliothèque que les délicieux cookies et plats végétariens issus de son potager, Françoise, malgré son handicap n'hésitait pas non plus à voyager jusqu'à la fin des années 2000, à l'invitation de Harvard et de l'UCLA. Et, au milieu de cette intense vie scientifique et sociale, Françoise trouvait encore, Dieu sait où, l'énergie d'être une citoyenne pleinement engagée dans de nombreuses causes humanitaires et civiques, des plus connues aux plus discrètes - et en sus, d'être une femme de grande culture qui trouve le temps d'aller au concert. Saluons enfin son humour dont elle s'est faite elle-même, souvent, la première cible. Dans son In memoriam de Walther Heissig décédé en 2005, elle écrivait «Voici brusquement les mongolisants devenus orphelins ${ }^{5}$ »; ils ont aujourd'hui perdu père et mère !

\section{Françoise Aubin}

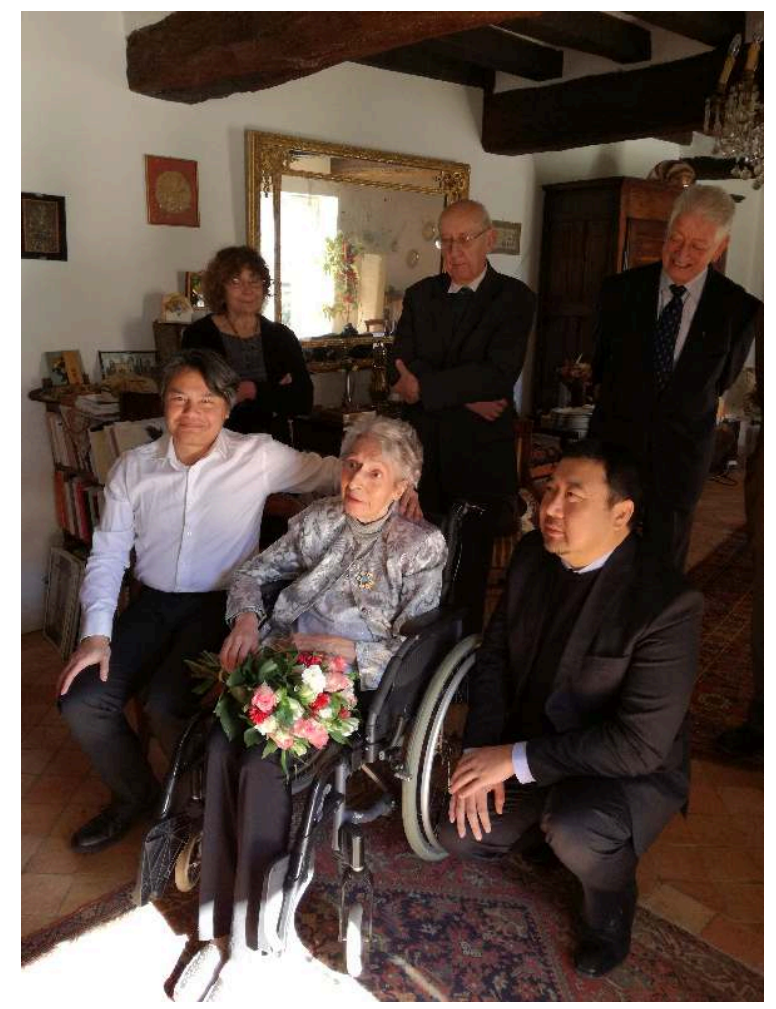

Monsieur M. Batsaikhan, ambassadeur de Mongolie en France, remet la médaille de l'Étoile Polaire à Françoise Aubin dans sa maison du Parc de Séronne le 18 février 2017.

(c) Isabelle Charleux (2017)

\section{BIBLIOGRAPHIE}

Bibliographie sélective de Françoise Aubin, ayant trait aux études mongoles

1960

Index de « Un Code des Yuan» de P. Ratchnevsky, in Mélanges publiés par l’Institut des Hautes Études 
chinoises 2 (Paris, Presses universitaires de France, Bibliothèque de l'Institut des Hautes études chinoises 14), pp. 423-515.

1965

Les soulèvements populaires au Shandong entre 1214 et 1230 , Thèse de doctorat. Prix de la meilleure thèse 1966 de la Faculté de droit de Paris (membres du jury : Profs Carbonnier, Duverger, Imbert). Paru en microfiches AUDIR, Fondation nationale des Sciences politiques, 1976. L'ancienne littérature mongole, reflet de la vie des steppes, Démocratie nouvelle, pp. 129-134. Deux poèmes d'aujourd'hui, Démocratie nouvelle, pp. 140-141.

1966

Une société d'émigrés : la colonie des Kalmouks de France, L'année sociologique 17, pp. 133-212.

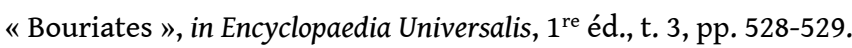

Francuzy o Sibirii [Les Français parlant de la Sibérie], Vostočno-Sibirskaja pravda 157/13792, $1^{\mathrm{er}}$ juillet 1966, p. 4.

Franc emegtej setgegdel [Impressions d'une Française], Utga zohiol urlag 50, 9 décembre 1966.

1967

Une expérience de collectivisation en économie nomade. La coopérative de production rurale en R.P.M., L'Homme et la Société 5(1), pp. 141-148.

Impressions d'automne et d'hiver dans la steppe mongole, Démocratie nouvelle juin.

Francuzy v Mongolii [Les Français en Mongolie], Sovremennaja Mongolija décembre, p. 22

(traduction mongole abrégée dans Orčin üjeijn Mongol uls décembre 1967).

1968

La Mongolie traditionnelle, Documentation pédagogique 154.

Novye gorizonty [Nouveaux horizons], Sovremennaja Mongolija 1968(4), p. 18.

en russe, une brève note sous la rubrique « O ljudjah, o zemle Mongolii glazami zarubežnyh

gostej » [Gens et terre de Mongolie vus par nos hôtes étrangers], Novosti Mongolii 52/5112, 3 juillet 1968 , p. 4.

1970

Les mesures manuelles et par référence au corps chez les Mongols. Note de folklore juridique, in L. Ligeti (dir.) Mongolian Studies (Budapest, Akadémiai Kiadó), pp. 23-55.

Traditions et mutations : sociologie actuelle de la Mongolie, Cahiers internationaux de Sociologie 49, pp. 83-110.

1972

Mongolie 1971. Revue de quelques faits et chiffres récents, Revue des Pays de l'Est 1972(1), pp. 127-140.

La Mongolie intérieure et les Mongols de Chine : éléments de bibliographie I, Études mongoles 3 , pp. 1-158.

« Mongolie (Histoire) » et « Mongolie (République populaire de) », in Encyclopaedia Universalis, vol. 11, pp. 239-261.

« Ö̈rats et Kalmouks », in Encyclopaedia Universalis, vol. 12, pp. 29-31.

Nécrologie : Le Père Antoine Mostaert (10 août 1881- 2 juin 1971), T'oung Pao 58(1-5), pp. 218-220.

1973

Fêtes et commémorations en République populaire de Mongolie. Apport à l'étude de la propagande éducative en pays socialiste, Revue française de Science politique 23(1), pp. 33-58. Les études mongoles en France, Studia Mongolica 1/9(1-17), pp. 198-200.

1974

Anthropologie du nomadisme, Cahiers internationaux de Sociologie 56, pp. 79-90. 
Spécificité culturelle et industrialisation en Mongolie, Revue tunisienne de Sciences sociales 36-39, pp. 245-277.

Nomadizm i sociologija sela [Nomadisme et sociologie rurale], in Rol' kočevyx narodov v civilizacii central'noj Azij [Le rôle des nomades dans les civilisations d'Asie centrale] (Ulaanbataar, Ak.N. MNR), pp. 233-236.

Sinologie mongole. État des études menées en République de Mongolie sur la Chine du $\mathrm{X}^{\mathrm{e}}$ au XIV siècle et les dynasties barbares, Sung Studies Newsletter 9, pp. 10-17.

Nécrologie : Vilmos Diószegi (2 mai 1923 - 22 juin 1972), T’oung Pao 60(4-5), pp. 328-333.

1975

Le statut de l'enfant dans la société mongole, in L'Enfant (Bruxelles, Éditions de la Librairie encyclopédique, Recueils de la Société Jean Bodin 35), pp. 459-599.

En collaboration avec J.-P. Accolas, Les produits laitiers en République populaire de Mongolie, Études mongoles 6, pp. 55-79 (et collaboration de supervision à l'ensemble de l'article de J.P. Accolas \& J.-P. Deffontaines, Les activités rurales en République populaire de Mongolie, pp. 7-98).

\section{6}

Cadenas et clef - Note d'ethno-linguistique mongole, in W. Heissig, J. R. Krueger, F. J. Oinas \&

E. Schütz (dirs), Tractata Altaica Denis Sinor sexagenario optime de rebus altaicis merito dedicata (Wiesbaden, Otto Harrassowitz), pp. 11-44.

Notes à une contribution de J.-P. Accolas \& J.-P. Deffontaines, Quelques données sur l'élevage du yak en République populaire de Mongolie, Ethnozootechnie 15, pp. 133-141.

1977

En collaboration avec P. Ratchnevsky, Un Code des Yuan, t. 3, Index (Paris, Presses universitaires de France, Bibliothèque de l'Institut des Hautes études chinoises 4).

Asie centrale. Peuples et langues (Quelques travaux occidentaux, 1972-1977), $1^{\text {re }}$ partie, L'année sociologique 28, pp. 373-394.

1978

En collaboration avec J.-P. Accolas \& J.-P. Deffontaines, Le lait et les produits laitiers en République populaire de Mongolie, Le Lait. Revue générale des questions laitières 63(575-576), pp. 276-286.

Cheval céleste et bovin chtonien, in R. Dor \& M. Nicolas (dirs), Quand le crible était dans la paille. Hommage à Pertev Naili Boratav (Paris, G.-P. Maisonneuve et Larose), pp. 37-63.

1980

« Être mongolisant en 1980, ou, La nouvelle école française d'études mongoles » (Conférence à l'Institut belge des hautes études chinoises, Bruxelles, année académique 1980-1981, séance inaugurale du 6 octobre 1980).

1982

Pensée romano-germanique et droit mongol contemporain. Le langage juridique en République populaire de Mongolie, Acta Orientalia Hungarica 36(1-3), pp. 17-27.

Une communauté rurale en Mongolie ?, in Les Communautés rurales, troisième partie, Asie et Islam (Paris, Dessain et Tolra, Recueils de la Société Jean Bodin pour l'histoire comparative des institutions 47), pp. 339-358.

Être mongolisant en 1980, ou, La nouvelle école française d'études mongoles, Année académique 1980-1981. Séance inaugurale du 6 octobre 1980 (Bruxelles, Institut belge des hautes études chinoises). Asie centrale et peuples mongols : à propos de quelques publications, 1974-1982, L'Ethnographie 78(86), pp. 103-121. 
1983

Cinq contributions à Dupeigne, B. (dir.) Mongolie-Mongolie. Traditions de la steppe (Paris, Musée de l'Homme, Catalogue de l'exposition du Musée de l'Homme, mars-mai 1983) : Les traditions dans la Mongolie en mutation, pp. 7-8 ; Quelques réflexions sur la Mongolie et les Mongols, pp. 9-11; Le nomade, son cheval, sa selle, pp. 28-29 ; La cuisine mongole ? Un code social codé, pp. 48-50 ; La connaissance de la Mongolie dans nos pays, pp. 59-60.

Un cahier de vocabulaire technique du R.P. A. De Moerloose, CICM, missionnaire de Scheut (Gansu septentrional, fin du XIX siècle) », Cahiers de Linguistique d'Asie orientale 12(2), pp. 103-117.

\section{4}

In memoriam. Le R.P. Henry Serruys (Ssu Lü-ssu 司律思), CICM (10 juillet 1911-16 août 1983), érudit sino-mongolisant, Monumenta Serica 36, pp. 556-624.

«Bouriates », in Encyclopaedia Universalis, $2^{\mathrm{e}}$ éd., vol. 3, pp. 919-920.

« Kalmouks », in Encyclopaedia Universalis, 2 éd., vol. 10, pp. 770-771.

«Oirat », in Encyclopaedia Universalis, $2^{\mathrm{e}}$ éd., vol. 13, pp. 432- 433 ;

« Mongolie (Histoire) », in Encyclopaedia Universalis, $2^{2}$ éd., vol. 12, pp. 528-549.

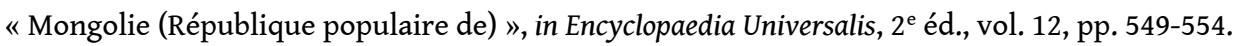

1985

À propos des Kalmak ou Sart-Kalmak de l'Issyk-kul', Études mongoles et sibériennes 16, pp. 81-89 + traduction (du russe) et adaptation de l'article de N.L. Zhukovskaya, Les Kalmak de l'Issyk-kul', pp. 91-106 (repris dans Études orientales 15-16, 1995).

À propos du père Henry Serruys, C.I.C.M. (1911-1983), Études mongoles et sibériennes 6, pp. 7-16 (et édition posthume de six articles du P. Serruys, pp. 17-79).

In memoriam Jadamžavyn Cevel (1902-1984), Études mongoles et sibériennes 6, pp. 117-120.

\section{6}

L'art du cheval en Mongolie, in Production pastorale et Société. Recherches sur l'écologie et l'anthropologie des sociétés pastorales (Paris, MSH/CNRS, Supplément à MSH Informations 19), pp. 129-149.

Le folklore comme mass media. L'exemple de la selle chez les Mongols, L'Ethnographie 82(98-99), pp. 119-144.

L'empire nomade des Mongols, in P. Vidal-Naquet (dir.), Le grand livre de l'histoire du monde, pp. 112-113 (article publié sans les corrections de l'auteur, réédition avec corrections en 1987, dans l'Atlas historique).

\section{7}

The Rebirth of Chinese Rule in Times of Troubles. North China in the Early Thirteenth Century, in S. R. Schram (dir.), Foundations and Limits of State Power in China (London/Hong Kong, School of Oriental and African Studies/The Chinese University Press), pp. 113-146.

A Note about the Spread of Chinese Literature amongst the Mongols, in C. Salmon (dir.), Literary Migrations. Traditional Chinese Fiction in Asia (17-20 $0^{\text {th }}$ Centuries) (Beijing, International Culture Publishing Corporation), pp. 209-212.

Édition du volume posthume d'Henry Serruys, The Mongols and Ming China. Customs and History (Londres, Variorum Reprints).

In memoriam Louis Ligeti (Ligeti Lajos, 1902-1987), Études mongoles et sibériennes 18, pp. 139-140. In memoriam Cendijn Damdinsüren (1908-1986), Études mongoles et sibériennes, 18, pp. 141-147. L'empire nomade des Mongols, (réédition, avec corrections) in P. Vidal-Naque (dir.), Atlas historique. Histoire de l'humanité, de la préhistoire à nos jours (Paris, Hachette), pp. 112-113. À propos de Joseph Kler, cicm (1897-1969), Cavaliers du désert mongol (Ordos), Production pastorale et Société 20, pp. 35-36; et édition de J. Kler, Cavaliers du désert mongol (Ordos), Production pastorale et Société 20, pp. 37-38. 
À propos de 'Missionary Safe-Conducts. An Additional Note' du P. Henry Serruys, cicm

(1911-1983), T'oung Pao 23, pp. 113-115.

1988

En collaboration avec R. Hamayon, L'individu face au pouvoir dans la Mongolie sous domination mandchoue ( $\mathrm{xVI}^{\mathrm{e}}-\mathrm{xx}$ siècles), in L'Individu face au pouvoir (Paris, Dessain et Tolra, Recueils de la Société Jean Bodin pour l'histoire comparative des institutions 47), pp. 321-329.

In memoriam Cendijn Damdinsüren (septembre 1908-juin 1986), Études mongoles et sibériennes 18, pp. 141-147.

Louis Ligeti (Ligeti Lajos, octobre 1902-mai 1987), Journal asiatique 276(1-2), pp. 1-22.

1989

La vision catholique de la religiosité chinoise et mongole. L'expérience des missionnaires de Scheut en Mongolie chinoise, Mélanges de l'École française de Rome. Italie et Méditerranée 101(2), pp. 991-1035.

En collaboration avec T. H. Hahn, A Bibliography of European non-English Works on Sung, Liao, Chin, Hsi-Hsia and Yüan, Bulletin of Sung Yuan Studies 21, pp. 115-148.

Religions et croyances de la Chine et de la Haute Asie, Archives de Sciences Sociales des religions 67(2), pp. 175-189.

Entre Ciel et Terre, l'idéal du cheval en Chine, in V. Courtot-Thibault (coordin.), Le Petit livre du cheval en Chine (Lausanne, Caracole/Favre), pp. 77-100.

Réédition, avec mise à jour, « Mongolie (histoire) », in Encyclopaedia Universalis, $3^{\mathrm{e}}$ éd., vol. 15, pp. 658-678

Réédition, avec mise à jour, « Mongolie (République populaire de ) » in Encyclopaedia Universalis,

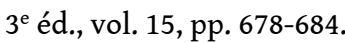

Réédition, avec mise à jour, « Bouriates ", in Encyclopaedia Universalis, $3^{\mathrm{e}}$ éd., vol. 14, pp. 455-456.

1990

Palanquin rouge et catholicisme. Le mariage chrétien en Mongolie chinoise (Missions belges de Scheut, 1865-1950), Neue Zeitschrift für Missionwissenschaft - Nouvelle Revue de Science missionnaire 46(2), pp. 81-98, 46(3), pp. 176-187.

Donneurs ou preneurs, quelques réflexions sur le jeu des transferts culturels entre Chinois, Turcs et Mongols, in R. Dor (dir.) L'Asie centrale et ses voisins. Influences réciproques (Paris, Inalco, Colloque Langues'O), pp. 165-180.

In memoriam le R.P. Joseph Van Hecken (Ho Ko-nan 賀歌南), C.I.C.M. (18 sept. 1905 - 31 août 1988), mongolisant, sinologue, missiologue, Monumenta Serica 39, pp. 325-350.

1991

Les sanctions et les peines chez les Mongols, in La Peine / Punishment, Quatrième partie, Mondes non européens/Non European Worlds (Bruxelles, De Boek Université, Recueils de la Société Jean Bodin pour l'histoire comparative des institutions 58), pp. 242-293.

1993

Co-édition des Cahiers d'études sur la Méditerranée orientale et le monde turco-iranien 16, numéro spécial Istanbul Oulan-Bator. Autonomisation, mouvements identitaires, construction du politique, pp. 135-204.

En collaboration avec J. Coussy \& S. Vaner, Conclusion, Cahiers d'études sur la Méditerranée orientale et le monde turco-iranien 16, pp. 351-372.

Renouveau gengiskhanide et nationalisme dans la Mongolie postcommuniste, Cahiers d'études sur la Méditerranée orientale et le monde turco-iranien 16, pp. 137-203.

La Mongolie, et, L'époque de Zanabazar, in G. Béguin \& D. Dashbaldan (dirs) Trésors de Mongolie, XVII ${ }^{e}$-XIX ${ }^{e}$ siècles. Musée national des Arts asiatiques - Guimet, Paris, 26 novembre 1993-14 mars 1994 (Paris, RMN), pp. 14-17 ; pp. 18-55, 238-239. 
Co-édition, avec contributions, Au Pays sacré des anciens Turcs. Exposition du Musée Guimet consacrée $\grave{a}$ H. de Bouillane de Lacoste (Paris, RMN).

Réédition de « Bouriates ", in Encyclopaedia Universalis, 4 e éd., vol. 4, pp. 455-456.

Écrits récents sur le Tibet et les Tibétains. Bibliographie commentée (Paris, Fondation nationale des Sciences politiques, Les Cahiers du CERI 6).

Contacts interethniques et notion d'ethnicité en République populaire de Chine, Revue bibliographique de Sinologie 11-12, pp. 151-152.

1994

La Mongolie des premières années de l'après-communisme : la popularisation du passé national dans les mass media mongols (1990-1995), in M.-D. Even (coordin.), Actes de la 37 $7^{e}$ P.I.A.C., Conférence internationale permanente des études altaïques, Chantilly, 20-24 juin 1994, Études mongoles et sibériennes 27, pp. 305-326.

Réédition, avec mise à jour, " Kalmouks ", in Encyclopaedia Universalis, $4^{\mathrm{e}}$ éd., vol. 13, pp. 248-249 Réédition, avec mise à jour, « Mongolie (histoire) », in Encyclopaedia Universalis, 4 éd., vol. 15, pp. 656-678.

Réédition, avec mise à jour, « Mongolie (République mongole) », in Encyclopaedia Universalis, $4^{\mathrm{e}}$ éd., vol. 15, pp. 678-684.

1995

À propos des Kalmak ou Sart-Kalmak de l'Issyk-kul', Études Orientales 15-16, pp. 73-79; traduction adaptée de l'article de N. L. Zhukovskaya, Les Kalmak de l'Issyk-kul', pp. 80-92 (repris de Études mongoles 16, 1985).

Réédition, avec mise à jour, « Oirat », in Encyclopaedia Universalis, 4 e éd., vol. 16, pp. 820-821.

\section{6}

La Mongolie des premières années de l'après-communisme : la popularisation du passé national dans les mass media mongols (1990-1995), Études mongoles et sibériennes 27, pp. 305-326.

Systèmes religieux et para-religieux en Chine et en Asie centrale (quelques publications, 1991-1995), Archives de Sciences Sociales des religions 93, pp. 129-158.

Reconstructions et langues voisines, Revue bibliographique de Sinologie 14, pp. 303-304.

1997

Sagesse des anciens, sagesse des enfants dans les steppes mongoles, in F. Blanchon (dir.) Enfances (Paris, Presses universitaires de Paris-Sorbonne, Asie 4), pp. 95-113.

Komünizm sonrası Moğolıstan'da Milliyetçilik ve Cengiz Hancı yenilenme [traduction turque de l'article : Renouveau gengiskhanide et nationalisme dans la Mongolie postcommuniste, Cahiers d'études sur la Méditerranée orientale et le monde turco-iranien 16, 1993], in S. Vaner (dir.), Unutkan Tarih Sovyet Sonrası Türkdili Alan (Istanbul, Metis Yayınları), pp. 135-202.

1998

Ou Falan 欧法蘭, Faguodi mengguxue yanjiu, 1949-1995 nian 法國的蒙古學研究 [Les études mongoles en France, 1949-1995], in J.-P. Drège (dir.) Faguo dangdai zhongguo-xue 法國當代中國學 [Cinquante ans d'études chinoises en France] (Beijing, Éditions de l'Académie des Sciences sociales), pp. 419-452. Article repris dans Mengguxue xinxi 蒙古學信息 [Nouvelles des études mongoles], 1998(1), pp. 26-43.

1999

Quelques aperçus sur la mort dans le catholicisme des Chinois et des Mongols (XIX $\mathrm{e}^{\mathrm{e}}$-première moitié du Xx ${ }^{\mathrm{e}}$ siècle), in P. Servais (dir.) La Mort et l'au-delà. Une rencontre de l'Orient et de l'Occident (Louvain-la-Neuve, Academia Bruylant, Rencontres Orient-Occident), pp. 197-239. Young Fr Mostaert's Forerunners, in K. Sagaster (dir.) Antoine Mostaert (1881-1971), C.I.C.M. Missionary and Scholar, vol. 1, Papers, (Louvain, Ferdinand Verbiest Foundation, Louvain Chinese 
Studies 4), pp. 31-45.

Une colonie étrangère d'un type nouveau à Oulan-Bator et dans les environs ( $1^{\text {re }}$ partie). Les prêtres et religieuses catholiques, Anda 32, pp. 2-5.

Une colonie étrangère d'un type nouveau à Oulan-Bator et dans les environs ( $2^{\mathrm{e}}$ partie). Les mouvements chrétiens non catholiques, Anda 33-34, pp. 5-6.

Critères d'appréciation des chevaux dans la tradition des nomades mongols, in Le Cheval en Eurasie. Pratiques quotidiennes et déploiements mythologiques (Paris, L'Harmattan, Eurasie 8), pp. 65-86.

2000

Quelques échos des prêtres chinois dans les missions de Scheut (Mongolie-Intérieure et Chine occidentale), dix-neuvième - vingtième siècles, in K. De Ridder (dir.), Footsteps in Deserted Valleys. Missionary Cases, Stategies and Practice in Qing China, (Louvain, Leuven University Press/Ferdinand Verbiest Foundation, Louvain Chinese Studies 8), pp. 161-184.

L'enquête sur le terrain par excellence : missionnaires de Scheut et études mongoles, Anda 39, pp. 10-12.

The Turco-Mongol Period, in M. A. Al-Bakhit, L. Bazin \& S. M. Cissoko (dirs), History of Humanity, Scientific and Cultural Development, vol. 4, From the Seventh to the Sixteenth Century (London/NewYork/Paris, Routledge/UNESCO), pp. 286-288.

2001

Mongolie d'autrefois. Premières observations des missionnaires catholiques de Scheut en Mongolie-Intérieure (1871), Anda 40, pp. 18-20.

\section{2}

Le déplacement absolu : le pastoralisme nomade des Mongols, in F. Blanchon (dir.), Aller et venir. Faits et perspectives (Paris, Presses de l'Université de Paris-Sorbonne, Asie 6-7), pp. 331-348. En collaboration avec Roberte Hamayon, Alexandre, César et Gengis-khan dans les steppes d'Asie centrale, in Les civilisations dans le regard de l'autre. Actes du colloque international, Paris, 13 et 14 décembre 2001 organisé conjointement par l'UNESCO et l'École pratique des Hautes Études, EPHE, dans le cadre de l'Année des Nations Unies pour le dialogue entre les civilisations (Paris, UNESCO, Dialogue entre les civilisations), pp. 73-106, 262-269.

Interrogation sur les droits humains : le cas de la Mongolie post-communiste, in T. Marrès \& P. Servais (dirs), Droits humains et valeurs asiatiques. Un dialogue est-il possible? (Louvain-la-Neuve, Academia Bruylant, Rencontre Orient-Occident 5), pp. 143-175.

Quand les mots racontent les objets. À propos du coussin et du contre-quartier de la selle mongole, Acta Orientalia Hungarica 55(1-3), pp. 29-41.

\section{4}

Some Characteristics of Penal Legislation among the Mongols $\left(13^{\text {th }}-21^{\text {st }}\right.$ Centuries) ", Central Asian Law : An Historical Overview. A Festschrift for the Ninetieth Birthday of Herbert Franke, W. Johnson \& I.F. Popova, eds, Lawrence (Ks) : Society for Asian Legal History, pp. 119-151.

Le Japon en terre d'islam chinois et au pays de Gengis-khan (fin XIX ${ }^{\mathrm{e}}$ siècle - début $\mathrm{Xx}^{\mathrm{e}}$ siècle), in Le Japon et l'islam. L'islam au Japon. Études orientales 21-22, pp. 36-79, 80-87.

\section{5}

In Memoriam. Walther Heissig (5 déc.1913-5 sept.2005), Études mongoles \& sibériennes, centrasiatiques \& tibétaines 36-37, pp. 464-469 [en ligne, URL : https://emscat.revues.org/1015, consulté le 29 novembre 2017].

\section{6}

« Reflections on the Fletcher Legacy. The 2006 Joseph Fletcher Memorial Lecture » (Conférence à Harvard, 26 octobre 2006, Inner Asia and Altaic Studies PhD Committee). 
2008

«Rambling Reflections on Mobility and Governability of Religious Phenomena and Systems in Inner Asia » (Conférence Mobility and Governability in Central Asia, à UCLA, 15 octobre 2008) «Law and colonial policies. Mongolian Penal Law and Sino-Manchu Control » (Conférence à USC, 20 octobre 2008).

2009

Written and Customary Law among the Mongols, in S. N. Katz (dir.) Encyclopaedia of Legal History (Oxford, Oxford University Press).

La loi, témoin de la vie. Les biens du nomade dans le droit pénal mongol (xVI $\mathrm{X}^{\mathrm{e}} \mathrm{XVIII}{ }^{\mathrm{e}}$ siècles, Zentralasiatische Studien - Festschrift Veronika Veit 38, pp. 21-52.

2010

To Impress the Seal. A technological Transfer, in I. Charleux, G. Delaplace \& R. Hamayon (dirs), Representing Power in Ancient Inner Asia. Legitimacy, Transmission and the Sacred (Bellingham, Western Washington University, Centre for East Asian Studies), pp. 159-207.

President Sambuu's Funeral in 1972, appendix to G. Delaplace, Marshall Choibalsan's Funerals, in I. Charleux, G. Delaplace \& R. Hamayon (dirs), Representing Power in Ancient Inner Asia. Legitimacy, Transmission and the Sacred (Bellingham, Western Washington University, Centre for East Asian Studies), pp. 117-122.

Préface, in L. Gardelle, Pasteurs nomades de Mongolie : des sociétés nomades et des États (Paris, BuchetChastel), pp. 13-16.

2011

Sinor Denis (17 avril 1916-12 janvier 2001), Études mongoles \& sibériennes, centrasiatiques \& tibétaines 36-37, pp. 464-469 [en ligne, URL : https://emscat.revues.org/1948 consulté le 29 novembre 2017].

2012

Jeux, fêtes et loisirs chez les nomades. Le cas de la Mongolie au cours du xx siècle, Anthropos 107(1), pp. 87-102.

Patrimoine artistique et réécriture du passé dans la Mongolie du Xxe siècle, Anthropos 107(1), pp. 467-479.

2013

En collaboration avec I. Bianquis \& S. Dulam, Le chien et la belle fille, in K. Buffetrille, J.-

L. Lambert, N. Luca \& A. de Sales (dirs), D'une anthropologie du chamanisme vers une anthropologie du croire. Hommage à l'œuvre de Roberte Hamayon, Études mongoles \& sibériennes, centrasiatiques \& tibétaines hors-série (Paris, CEMS/EPHE), pp. 303-321.

2015

En collaboration avec A. B. Bagader, Vues sur les Tatars de la Volga (Paris, L'Harmattan).

\section{NOTES}

1. Citons en particulier le $n^{\circ} 16$, dans lequel Françoise Aubin présente le Père Henry Serruys, et édite six de ses articles à titre posthume.

2. Elle y est stagiaire dès 1954, attachée de recherche de 1959 à 1966, chargée de recherche de 1966 à 1975, directeur de recherches de 1975 à 1997, puis directeur de recherches émérite de 1997 à 2006.

3. Pour sa bibliographie jusqu'en 2009, voir Miscellanea Asiatica.

4. En 1985, elle avait reçu à l'ambassade de Mongolie la médaille de l'Amitié Nairamdal.

5. https://emscat.revues.org/1015, consulté le 29 novembre 2017. 